\title{
Effect of Sex and Age on Repetitive, Stereotyped, and Exploratory Behaviors in a Rat Model of Valproic Acid-Induced Autism
}

\author{
Samaneh Rafiei ${ }^{1}$, Mohammad Amin Edalatmanesh ${ }^{2 *}$, Elahe Rezaei ${ }^{2}$ \\ ${ }^{1}$ Department of Exercise Physiology, Kish International Campus, Tehran University, Kish, Iran \\ ${ }^{2}$ Department of Physiology, College of Sciences, Shiraz Branch, Islamic Azad University, Shiraz, Iran
}

\section{A BSTRACT}

Introduction: Autism is characterized by behavioral impairments in social interaction, language and communication as well as restricted interests and activities. The present study examined stereotypical repetitive behaviors, anxiety, and locomotor as well as exploratory activities in rat model of valproic acid-induced autism. Materials and Methods: Pregnant Spraque dawley rats were administered by either valproic acid (VPA group, $500 \mathrm{mg} / \mathrm{kg}$, $\mathrm{n}=10$ ) or saline (control group, $\mathrm{n}=10$ ) during fetal neural tube development on embryonic day 12.5. All male or female offspring were investigated in this study. Stereotypical and anxietyexploratory behaviors were assessed using Y-maze and open field tests, respectively. Results: A single intra-peritoneal injection of VPA to pregnant rats produced severe autistic-like symptoms in the offspring. The results showed significant behavioral impairments including enhanced stereotyped repetitive behaviors, increased anxiety, and decreased exploratory activities in male rats at postnatal day 30 and 60 compared to female rats. Conclusion: Our data suggest that early embryonic exposure to VPA in rats provides an appropriate model for autism in male offspring.

Key words:

1. Autistic Disorder

2. Valproic Acid

3. Exploratory Behavior

*Corresponding Author: Mohammad Amin Edalatmanesh

E-mail:amin.edalatmanesh@gmail.com 


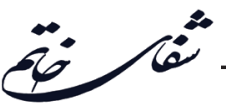

تأثير جنسيت و سن بر رفتارهاى تكرارى، كليشهاى و اكتشافى در مدل اوتيسم القاء شده با واليروئيك

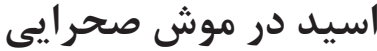

$$
\begin{aligned}
& \text { سمانه رفيعى'، محمد امين عدالت منش 'ّ، الهه رضايى' } \\
& \text { 'كروه فيزيولوزى ورزشى، برديس بين المللى كيش، دانشعاه تهران، كيش، ايران }
\end{aligned}
$$

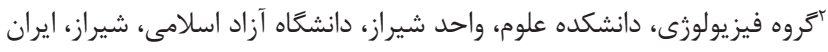

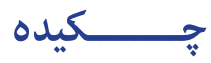

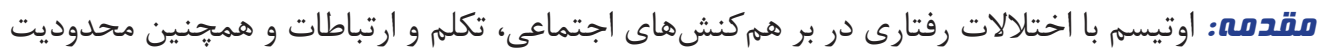

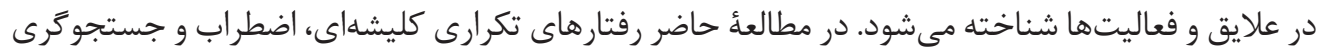

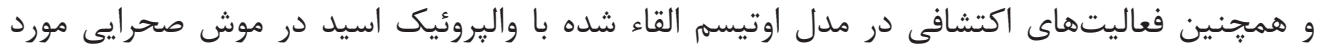

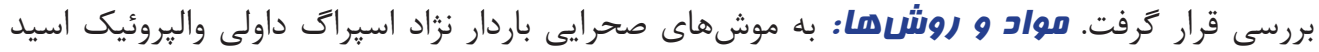

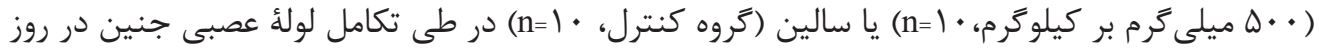

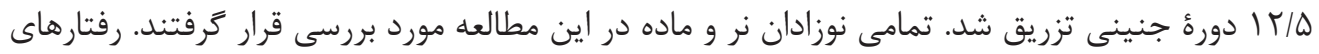

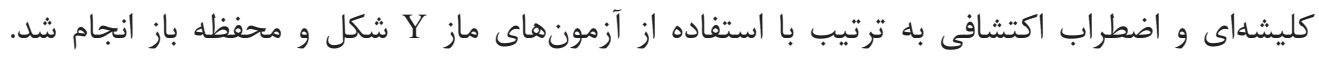

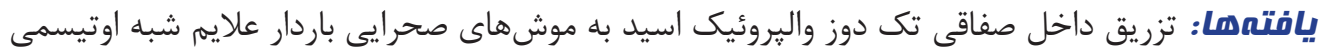

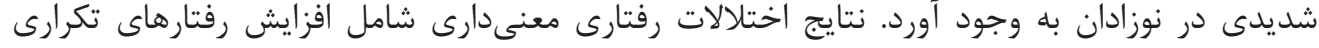

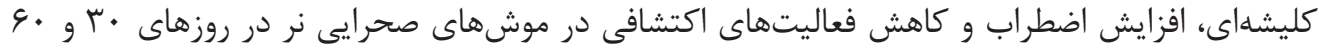

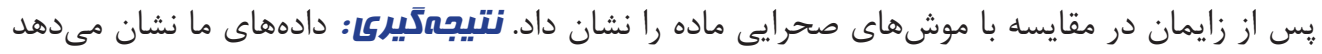

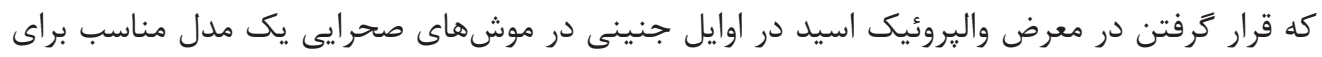
اوتيسم در نوزادان نر فراهم مى كنى دند.

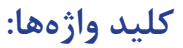

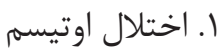

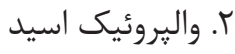

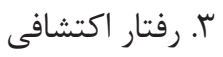

$$
\text { " نويسنده مسئول: محمد امين عدالت منش }
$$
آدرس الكترونيكى: amin.edalatmanesh@gmail.com 


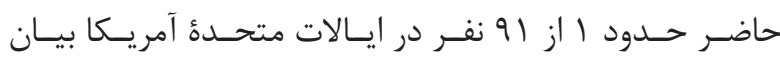

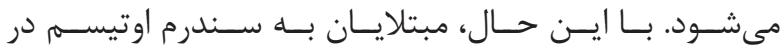

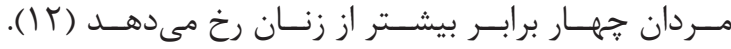

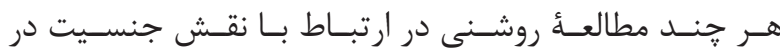

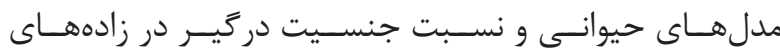

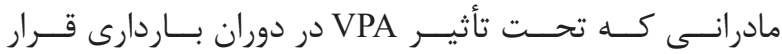

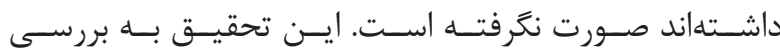

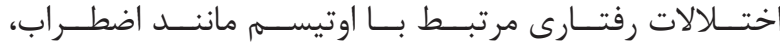

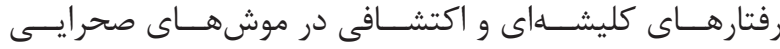

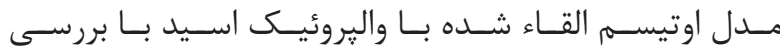

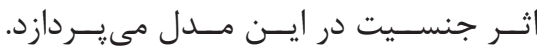

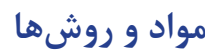

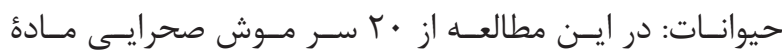

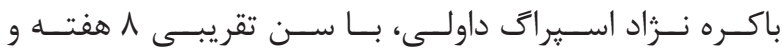

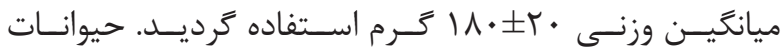

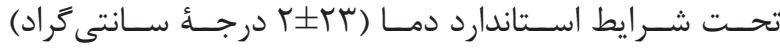

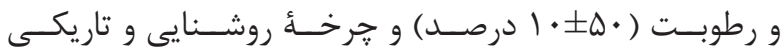

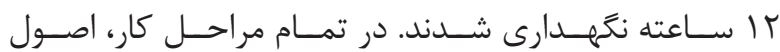

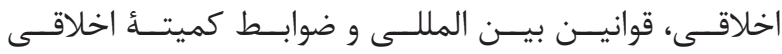

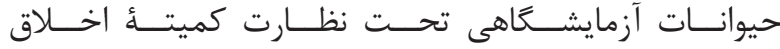

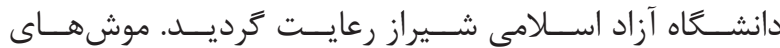

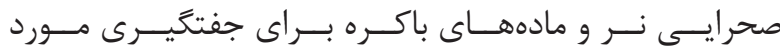

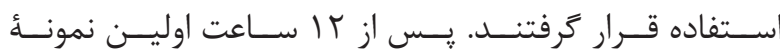

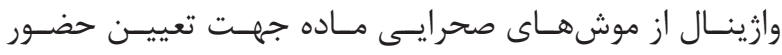

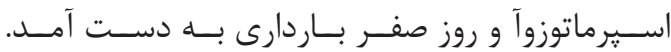

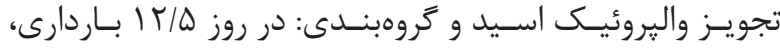

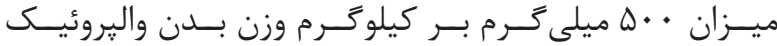

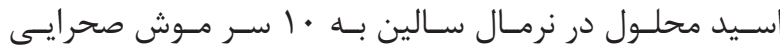

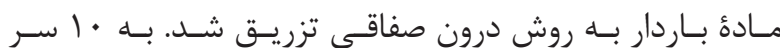

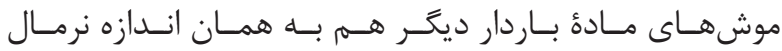

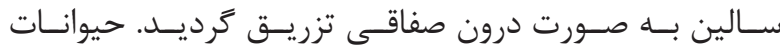

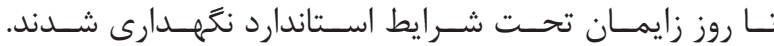

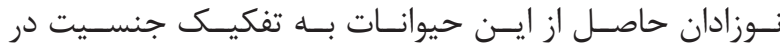

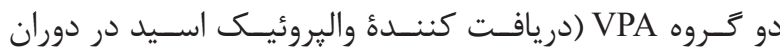

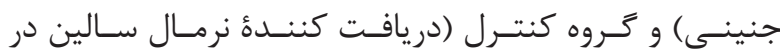

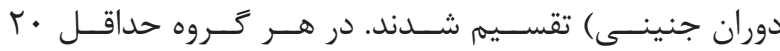

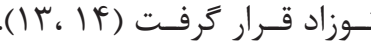

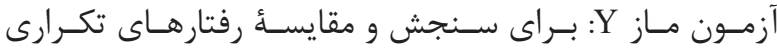

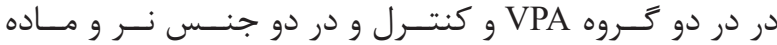

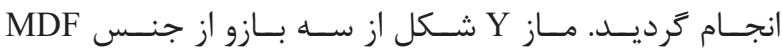

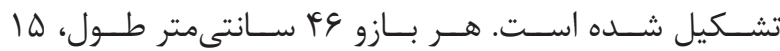

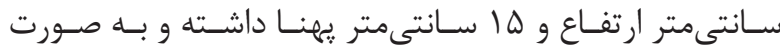

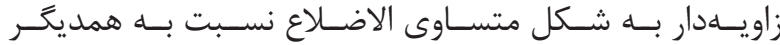

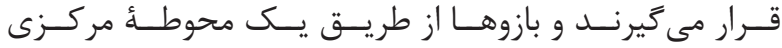

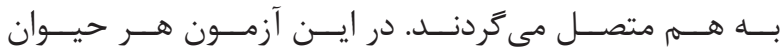

مقدمه

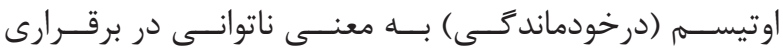

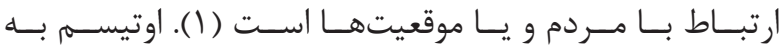

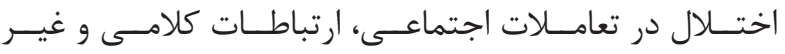

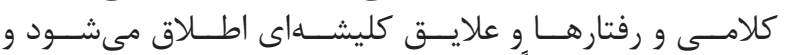

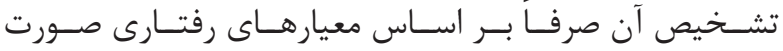

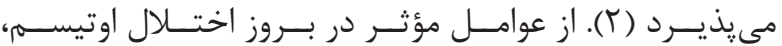

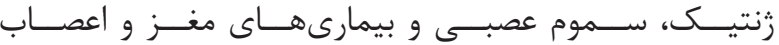

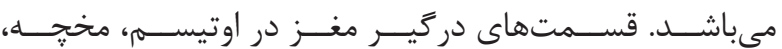

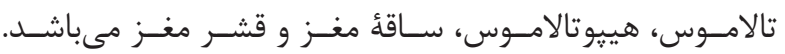

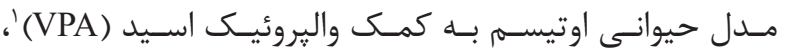

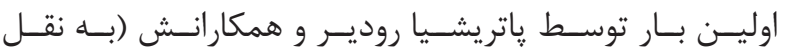

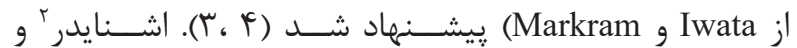

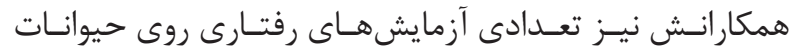

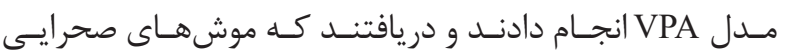

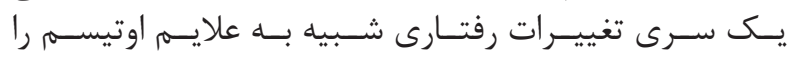

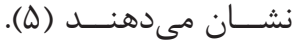

نقايـص اجتماعـى و ارتباطـى در بيمـارى اوتيسـم عبارتنـد ازي

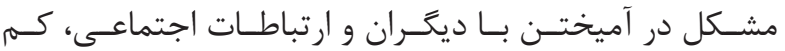

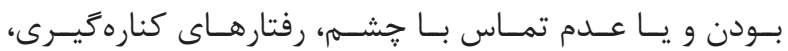

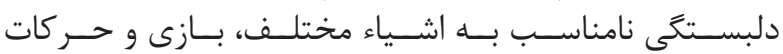

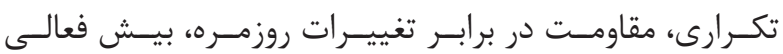

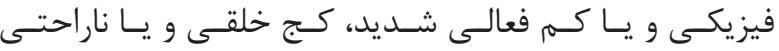

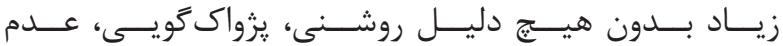

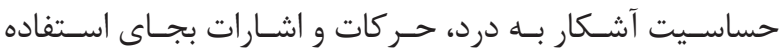

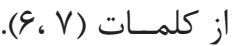

ارزش مدلهـــاى حيوانــى در نشــان دادن علايــم خـــاص و

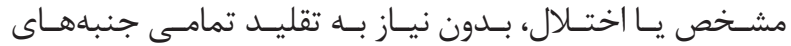

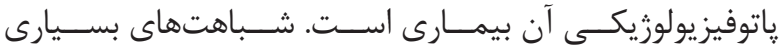

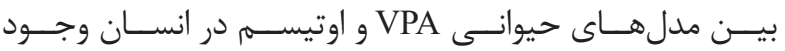

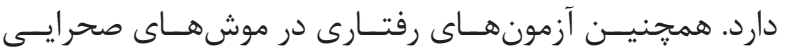

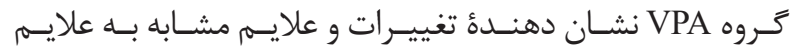

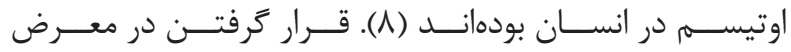

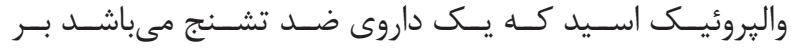

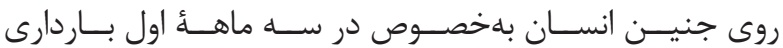

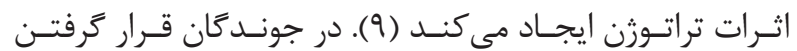

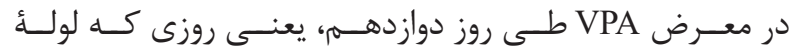

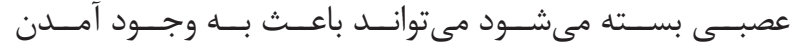

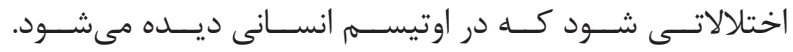

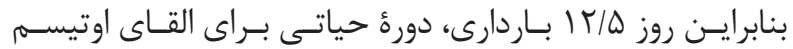

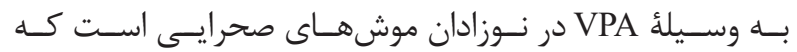

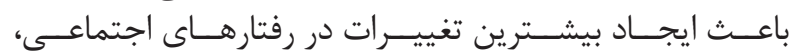

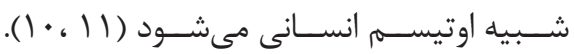

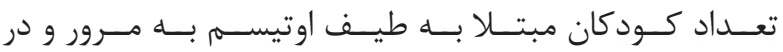

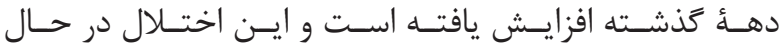




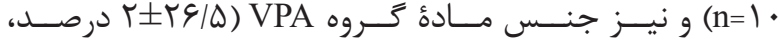

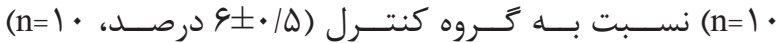

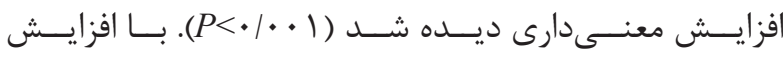

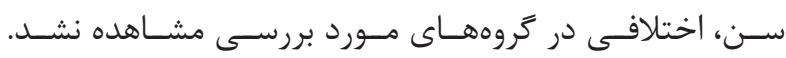

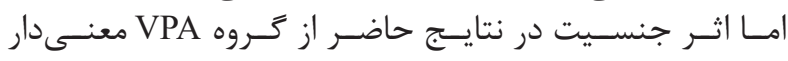

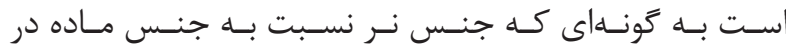

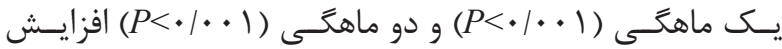

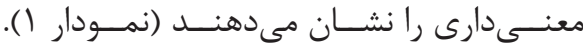

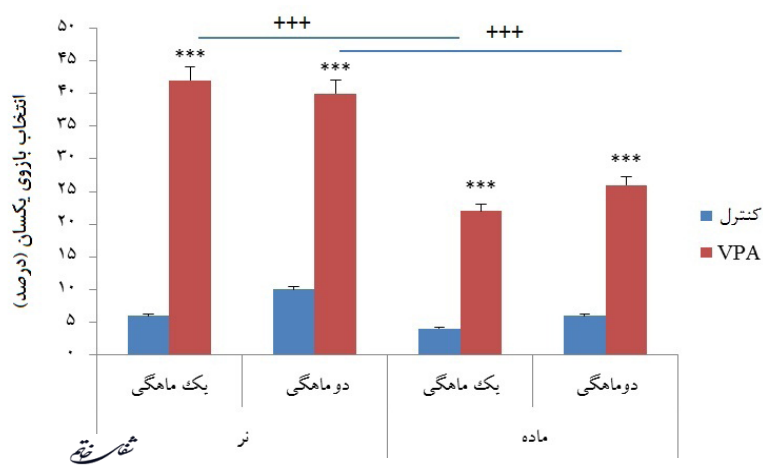

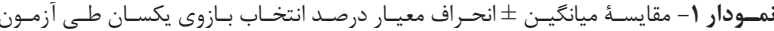

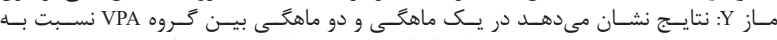

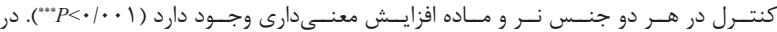

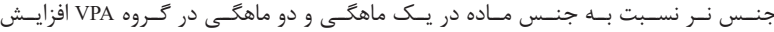

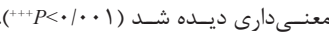

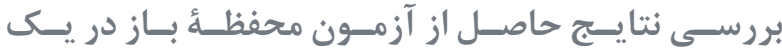

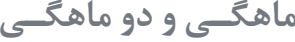

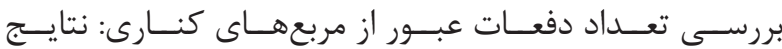

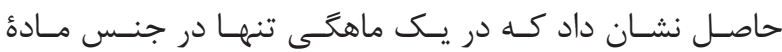

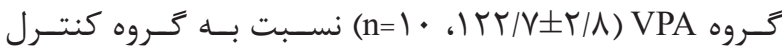

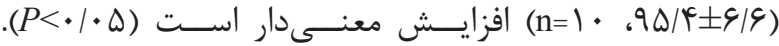

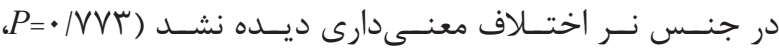

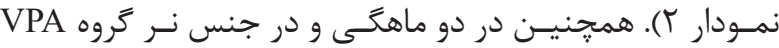

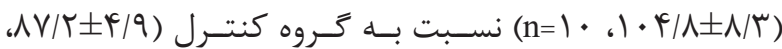

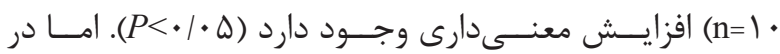

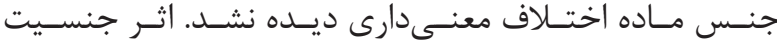

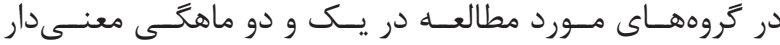

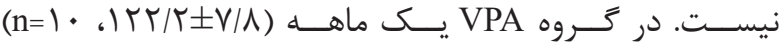

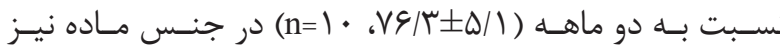

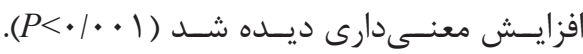

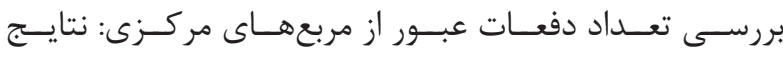

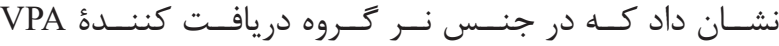

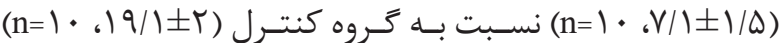

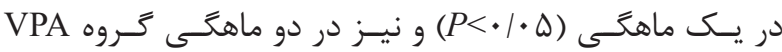

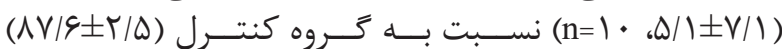

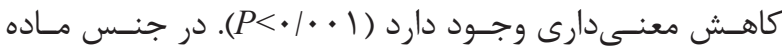

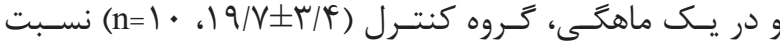

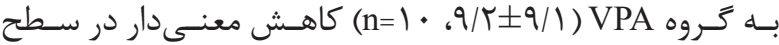

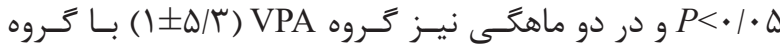

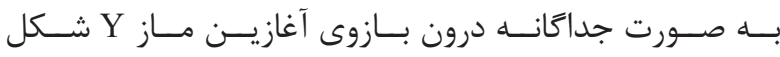

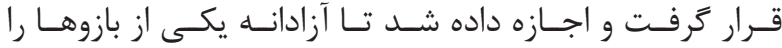

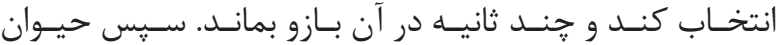

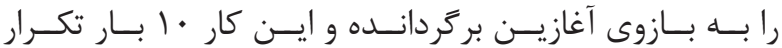

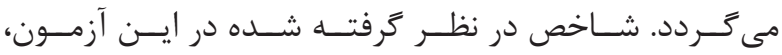

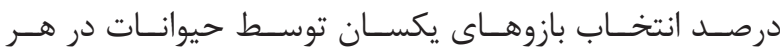

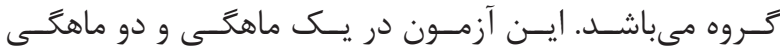

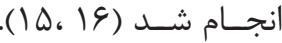

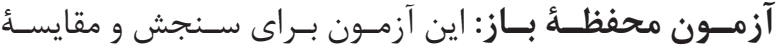

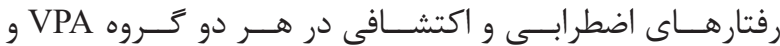

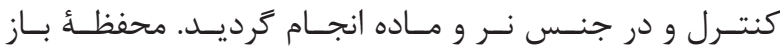

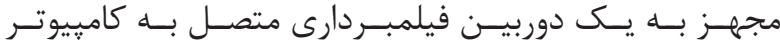

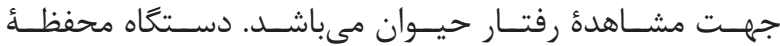

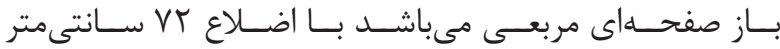

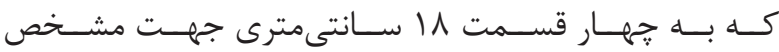

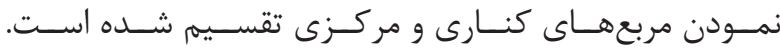

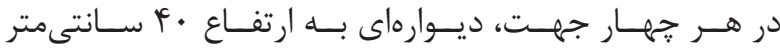

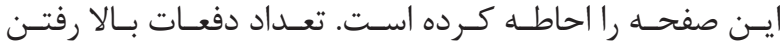

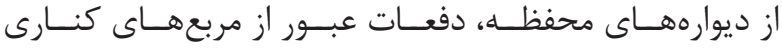

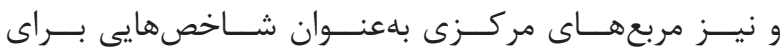

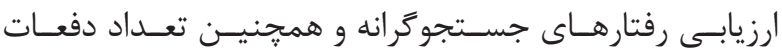

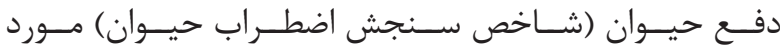

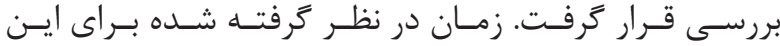

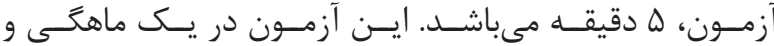

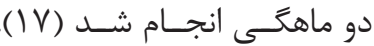

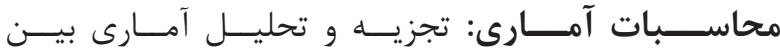

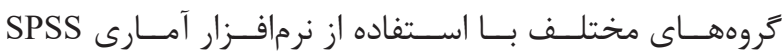

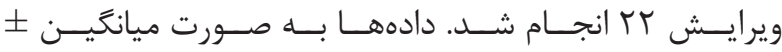

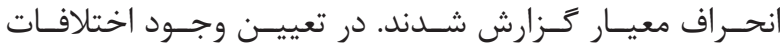

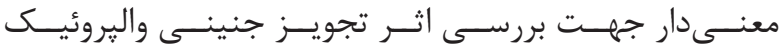

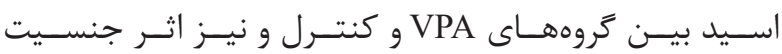

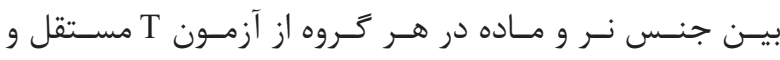

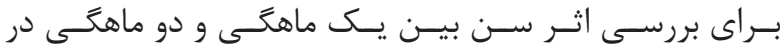

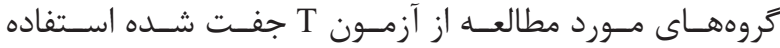

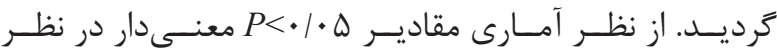

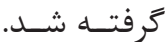

يافته ها

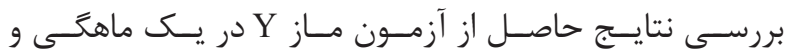

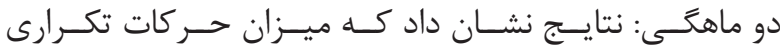

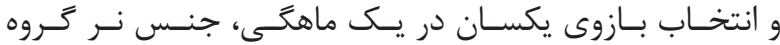

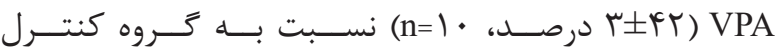

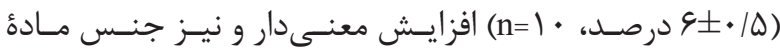
حـروه VPA

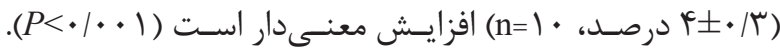

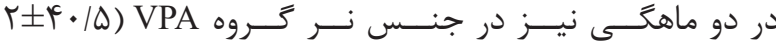

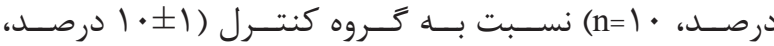




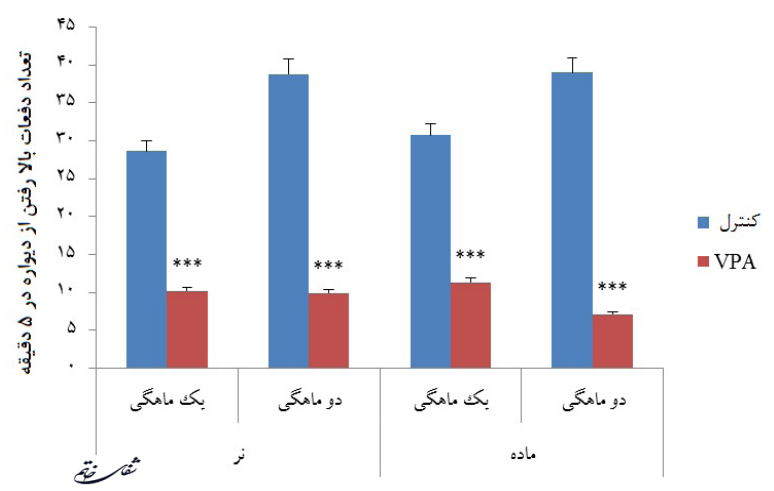

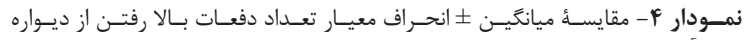

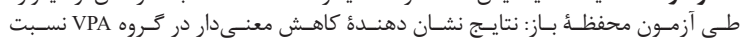

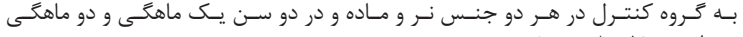

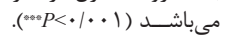

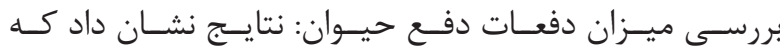

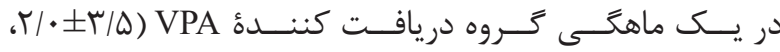

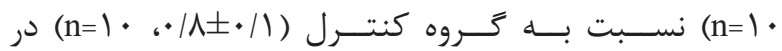

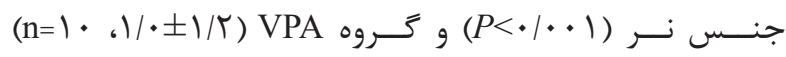

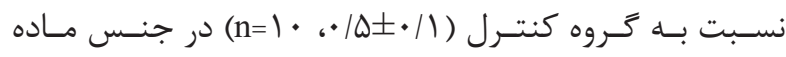

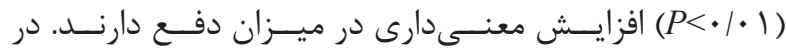

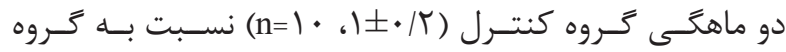

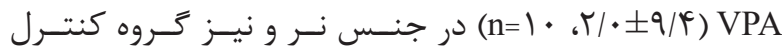

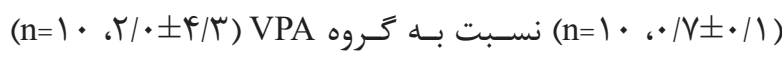

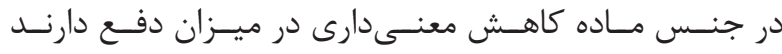
كاهـ

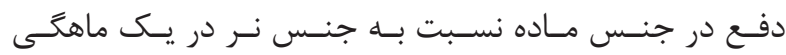

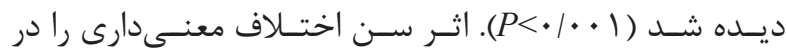

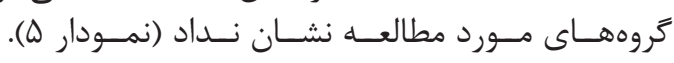

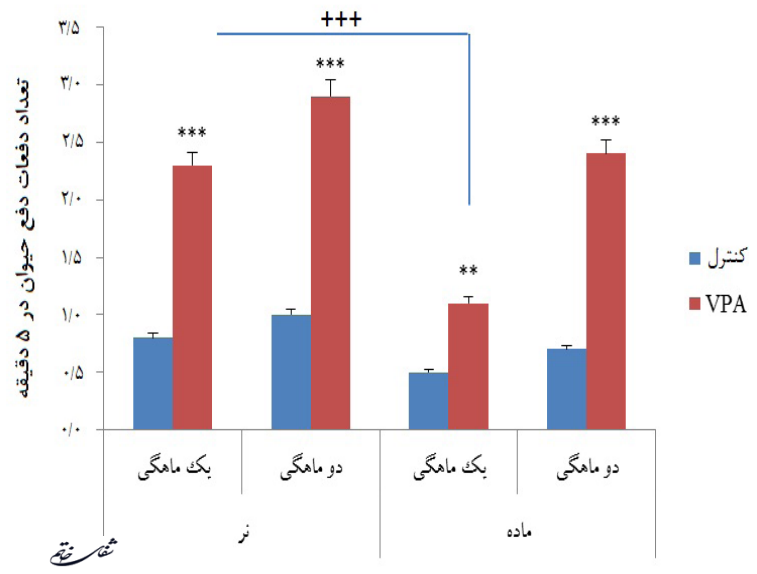

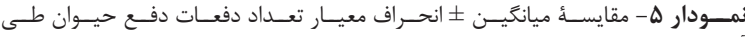

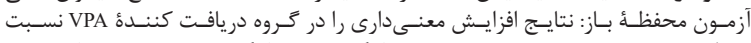

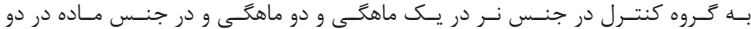

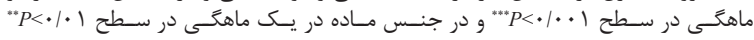

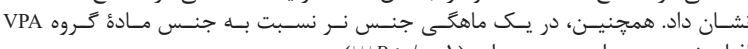

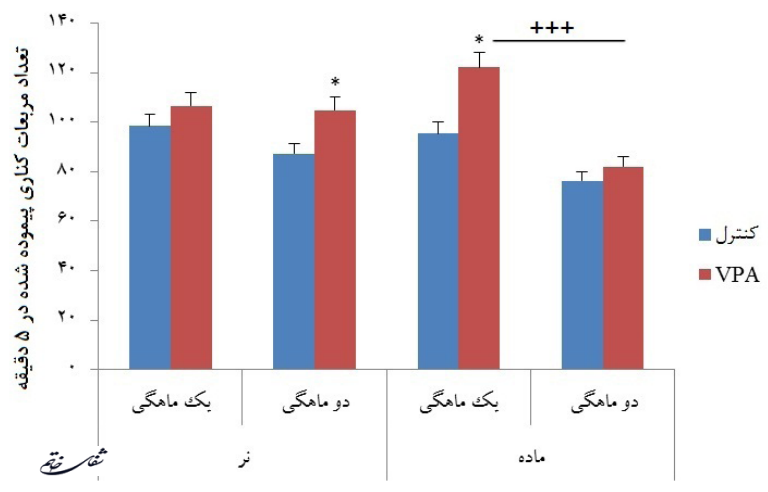

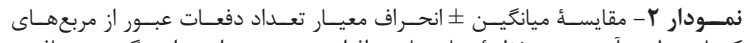

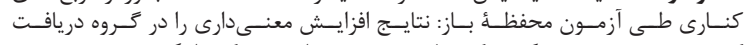

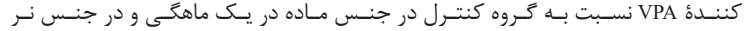

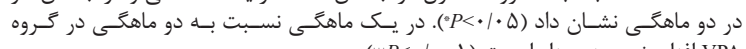

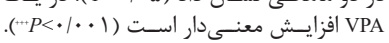

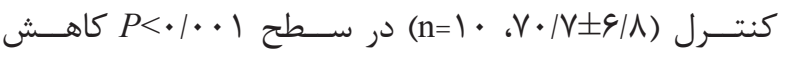

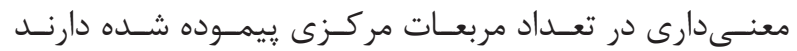

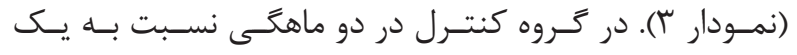

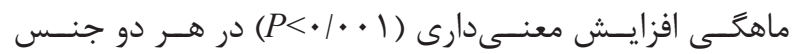
ديـده شـد.

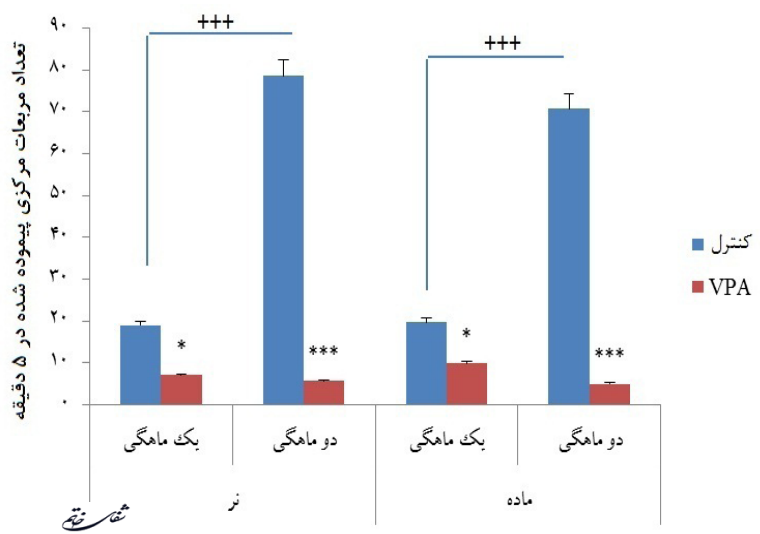

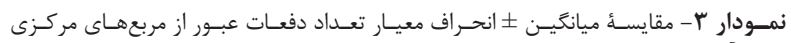

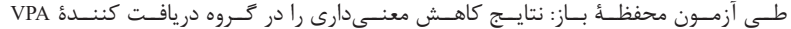

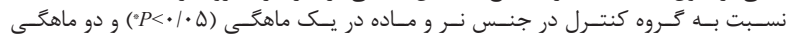

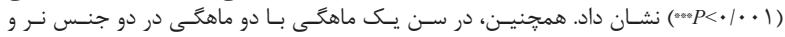

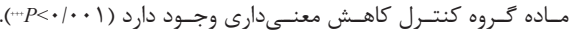

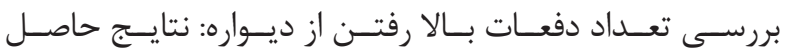

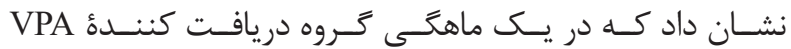

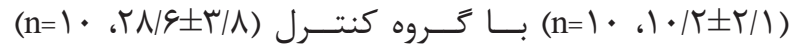

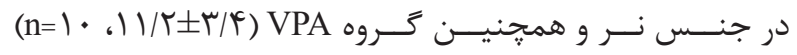

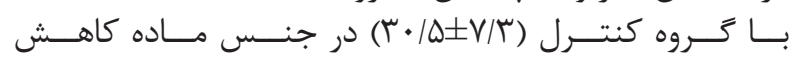

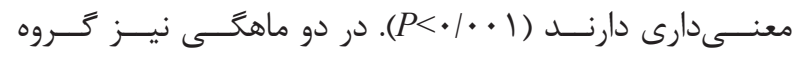

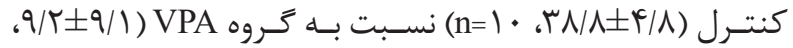

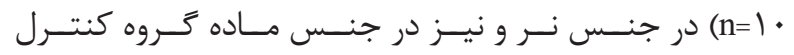

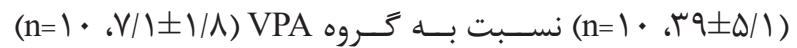

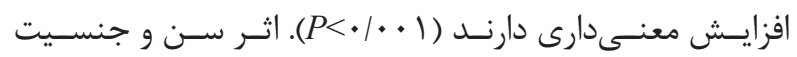
در هيـج تروهـى معنسى دار نبـود (نمــودار أ). 


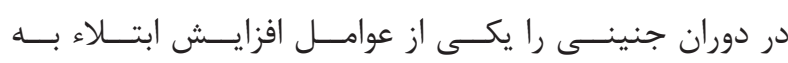

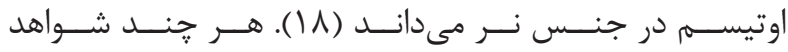

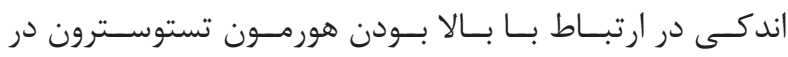

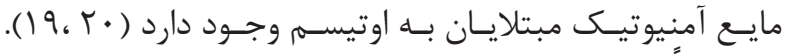

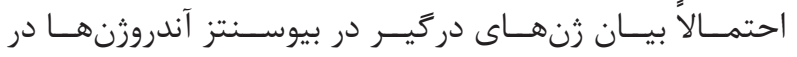

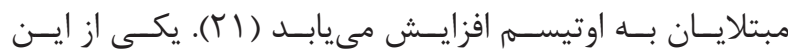

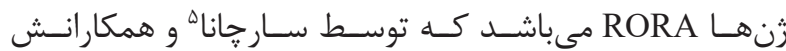

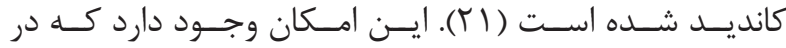

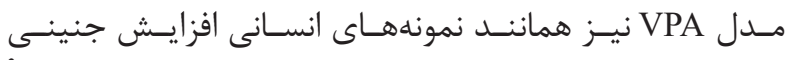

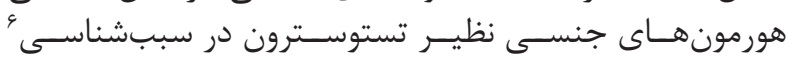

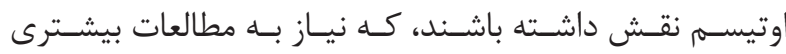
در ايـن زمينـهـ وجــود دارد.

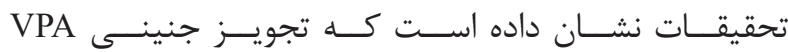

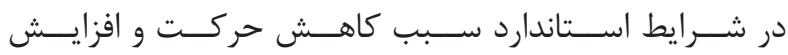

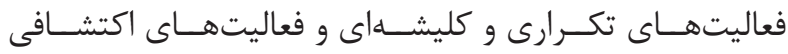

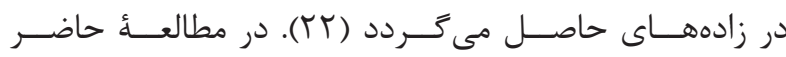

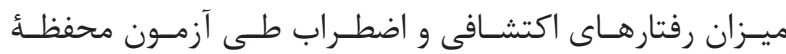

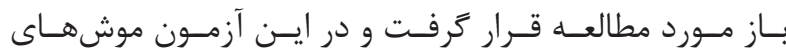

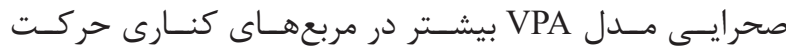

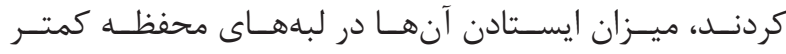

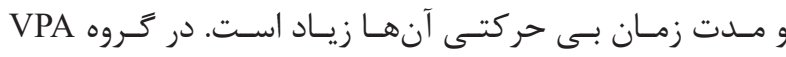

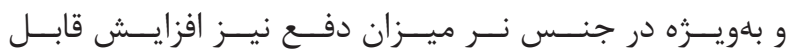

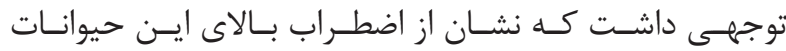

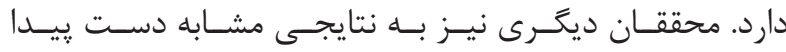

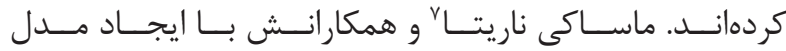

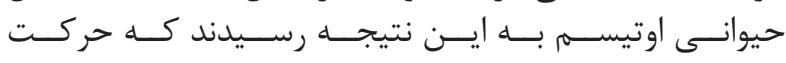

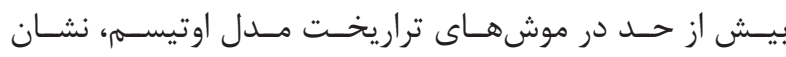

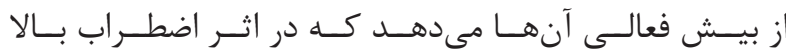

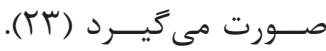

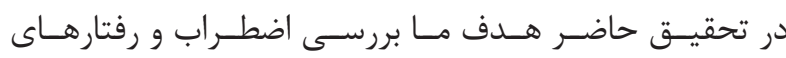

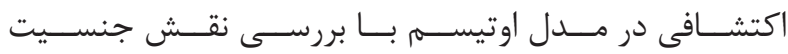

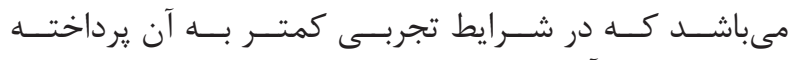

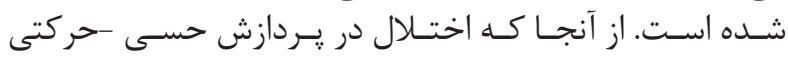

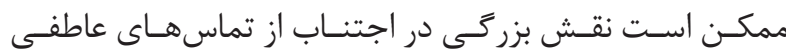

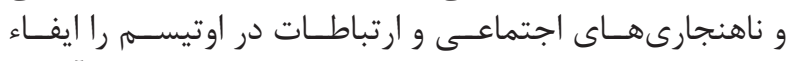

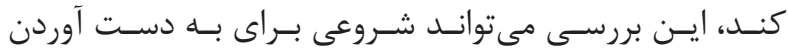

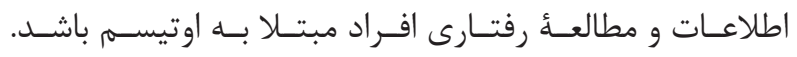

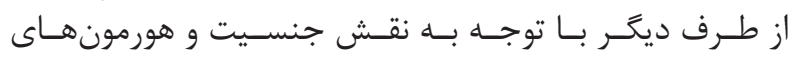

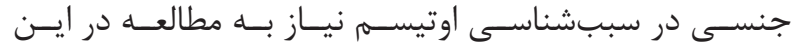
زمينسه وجــود دارد.

\footnotetext{
${ }^{3}$ Markram

${ }^{4}$ Baron-Cohen

${ }^{5}$ Sarachana
}

بحث و نتيجه

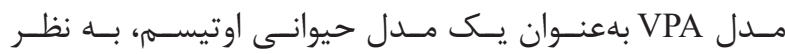

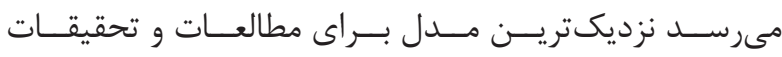

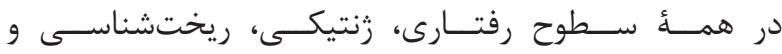

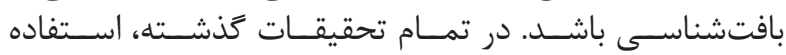

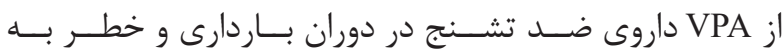

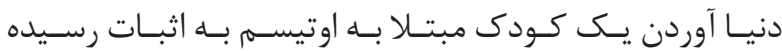

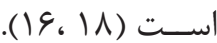

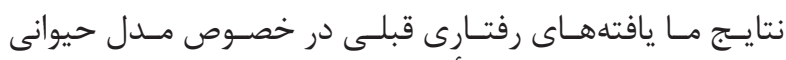

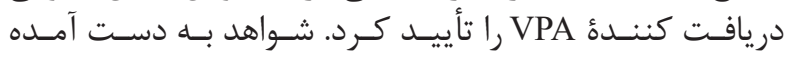

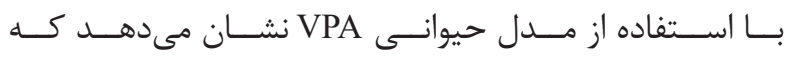

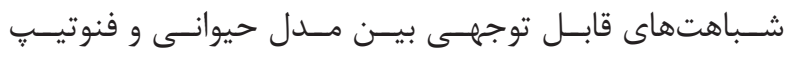

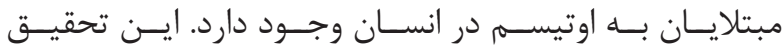

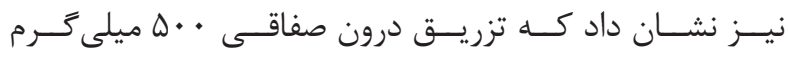

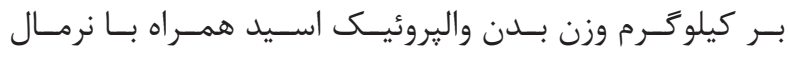

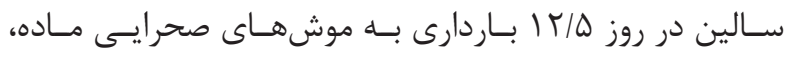

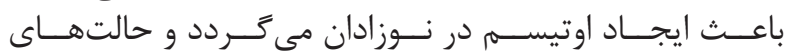

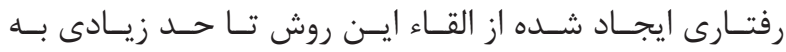

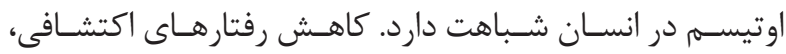

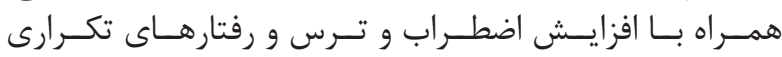

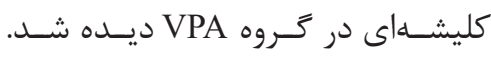

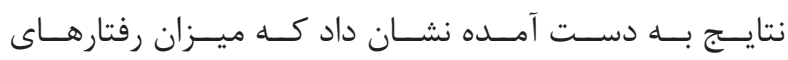

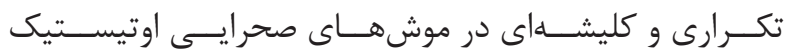

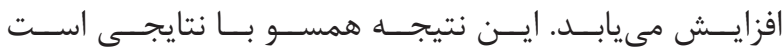

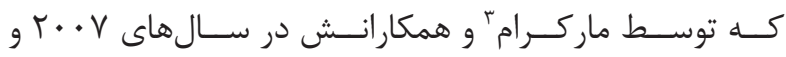

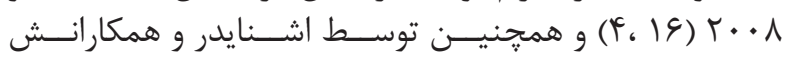

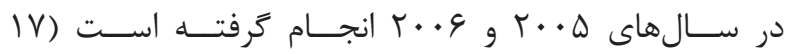

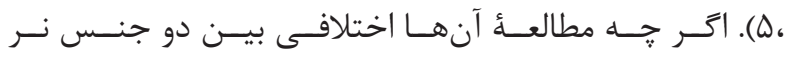

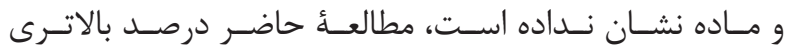

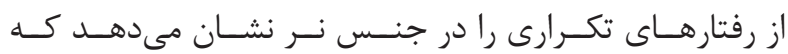

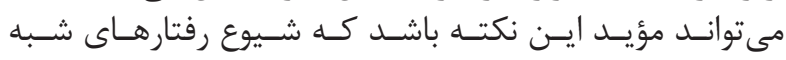

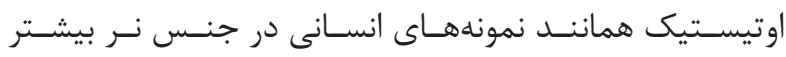

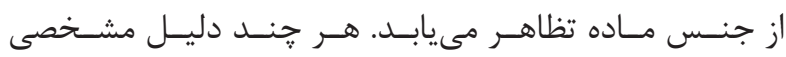

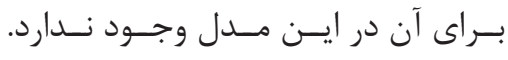

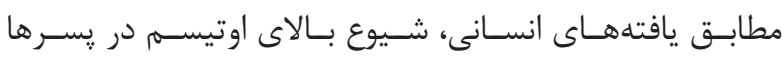

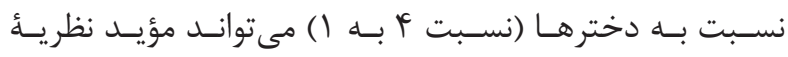

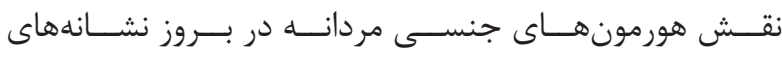

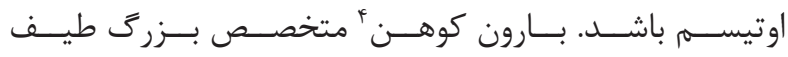

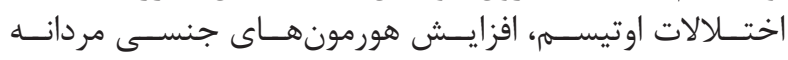

${ }^{6}$ Etiology
${ }^{7}$ Masaaki Narita 
1. Divanbeigi A, Divanbeigi A. A brief review on the causes of autism spectrum disorder. Shefaye Khatam. 2015; 3(1): 157-16.

2. Goldson E. Advances in Autism-2016. Adv Pediatr. 2016; 63(1): 333-55.

3. Iwata K, Matsuzaki H, Takei N, Manabe T, Mori N. Animal models of autism: an epigenetic and environmental viewpoint. J Cent Nerv Sys Dis. 2010; 2: $37-44$.

4. Markram H, Rinaldi T, Markram K. The intense world syndrome - an alternative hypothesis for Autism. Front Neurosci. 2007; 1(1): 77-96.

5. Schneider T, Turczak J, Przewłocki R. Environmental enrichment reverses behavioral alterations in rats prenatally exposed to valproic acid: issues for a therapeutic approach in autism. Neuropsychopharmacol. 2005; 31(1): 36-46.

6. Duch W, Nowak W, Meller J, Osinski G, Dobosz $\mathrm{K}$, Mikotajewski D, et al. Computational approach to underestanding Autism spectrum disorders. Comp Science. 2011; 13(2): 87-100.

7. Lynch CJ, Uddin LQ, Supekar K, Khouzam A, Phillips J, Menon V. Default mode network in childhood Autism: posteromedial cortex heterogeneity and relationship with social deficits. Biol Psychiatry. 2013; 74(3): 212-9.

8. Halladay AK, Amaral D, Aschner M, Bolivar VJ, Bowman A, DiCicco-Bloom E, et al. Animal models of autism spectrum disorders: information for neurotoxicologists. Neurotoxicol. 2009; 30(5): 811-21.

9. Duncan S. Teratogenesis of sodium valproate. Curr Opin Neurol. 2007; 20(2): 175-80.

10. Borzou Z, Edalatmanesh MA. The evaluation of brain derived neurotrophic factor and working memory in valproic acid animal model of Autism. Shefaye Khatam. 2015; 3(4):10-6.

11. Kim SJ, Silva RM, Flores CG, Jacob S, Guter S, Valcante $\mathrm{G}$, et al. A quantitative association study of SLC25A12 and restricted repetitive behavior traits in Autism spectrum disorders. Mol Autism. 2011; 2(1): 8. doi: 10.1186/2040-2392-2-8.

12. Dudova I, Kasparova M, Markova D, Zemankova J, Beranova S, Urbanek T, et al. Screening for autism in preterm children with extremely low and very low birth weight. Neuropsychiatr Dis Treat. 2014; 11: 10: 277-82.
منابع

13. Bambini-Junior V, Rodrigues L, Behr GA, Fonseca Moreira JC, Riesgo R, Gottfried C. Animal model of Autism induced by prenatal exposure to valproate: behavioral changes and liver parameters. Brain Res. 2011; 14(8): 8-16.

14. Damke E, Storti-Filho A, Irie MMT, Carrara MA, Batista MR, Donatti L, et al. Ultrastructural imaging of candida albicans adhesion to rat genital epithelium through scanning and transmission electron microscopy. Microsc Microanal. 2010; 16(3): 337-45.

15. Olexova L, Senko C, Štefanik P, Talarovicova A, Krskova L. Habituation of exploratory behaviour in VPA rats: animal model of Autism. Interdiscip Toxicol. 2013; 6(4): 222-7.

16. Markram K, Rinaldi T, La Mendola D, Sandi C, Markram H. Abnormal fear conditioning and amygdala processing in an animal model of Autism. Neuropsychop. 2008; 33: 901-12.

17. Schneider CK, Melmed RD, Barstow LE, Enriquez FJ, Ranger-Moore J, Ostrem JA. Oral human immunoglobulin for children with autism and gastrointestinal dysfunction: a prospective, open-label study. J Autism Dev Disord. 2006; 36(8): 1053-64.

18. Baron-Cohen S, Knickmeyer RC, Belmonte MK. Sex differences in the brain: implications for explaining Autism. Science. 2005; 310(5749): 819-23.

19. Auyeung B, Taylor K, Hackett G, Baron-Cohen S Foetal testosterone and autistic traits in 18 to 24-monthold children. Molecular Autism. 2010; 1(1): 11. doi: 10.1186/2040-2392-1-11.

20. Auyeung B, Baron-Cohen S, Ashwin E, Knickmeyer R, Taylor K, et al. Fetal testosterone and Autistic traits. Br J Psychol. 2009; 100(1): 1-22.

21. Sarachana T, Xu M, Wu RC, Hu VW. Sex hormones in Autism: androgens and estrogens differentially and reciprocally regulate RORA, a novel candidate gene for autism. PLoS One. 2011; 6(2): e17116.

22. Wagner GC, Reuhl KR, Cheh M, McRae P, Halladay AK. A new neurobehavioral model of autism in mice: pre-and postnatal exposure to sodium valproate. J Autism Dev Disord. 2006; 36(6): 779-93.

23. Narita M, Oyabu A, Imura Y, Kamada N, Yokoyama $\mathrm{T}$, Tano $\mathrm{K}$, et al. Nonexploratory movement and behavioral alterations in a thalidomide or valproic acidinduced autism model rat. Neurosci Res. 2010; 66: 2-6. 